\title{
The Mutational Meltdown in Asexual Populations
}

\author{
M. Lynch, R. Bürger, D. Butcher, and W. Gabriel
}

Loss of fitness due to the accumulation of deleterious mutations appears to be inevitable in small, obligately asexual populations, as these are incapable of reconstituting highly fit genotypes by recombination or back mutation. The cumulative buildup of such mutations is expected to lead to an eventual reduction in population size, and this facilitates the chance accumulation of future mutations. This synergistic interaction between population size reduction and mutation accumulation leads to an extinction process known as the mutational meltdown, and provides a powerful explanation for the rarity of obligate asexuality. We give an overview of the theory of the mutational meltdown, showing how the process depends on the demographic properties of a population, the properties of mutations, and the relationship between fitness and number of mutations incurred.

Although data are available for only a small number of species (Bell 1988b; Charlesworth et al. 1990; Crow and Simmons 1983; Houle et al. 1992; Lynch and Gabriel 1990; Mukai 1979), it now seems clear that deleterious mutations arise at a high rate in most organisms. Summing over all loci, and to an order of magnitude, the deleterious mutation rate appears to be at least one per diploid genome per generation, and indirect evidence suggests it could be much higher (Kondrashov 1988). The effects of these mutations are such that, in the absence of natural selection, an approximately $1 \%$ reduction in fitness would be expected per generation. Since practical limitations ensure that only a fraction of mutations are actually detected in empirical studies, these are likely to be underestimates of the deleterious mutation pressures that confront most organisms.

Natural selection, combined with re-

From the Department of Biology, University of Oregon, Eugene, OR 97403 (Lynch and Butcher), the Institut für Mathematik, Universität Wien, Vienna, Austria (Bürger), and the Department of Physiological Ecology, Max Planck Institute for Limnology, Plön, Germany (Gabriel). This paper was delivered at a symposium titled "The Evolution of Sex" sponsored by the American Genetic Association at Virginia Polytechnic Institute and State University in Blacksburg, Virginia, on July 10 and 11,1992 . We are very grateful to Brian Charlesworth, Thomas Hansen, David Houle, Alex Kondrashov, Russell Lande, and John Willis for several comments that helped clarify ideas in this paper. The work has been supported by NSF grants BSR 8911038 and BSR 9024977, and PHS grant GM36827 to M.L., a research exchange grant from the Max Kade Foundation to R.B., an NSF Genetic Mechanisms of Evolution training grant fellowship to D.B., and a grant from the Deutsche Forschungsgemeinschaft to W.G.

Journal of Heredity 1993;84:339-344; 0022-1503/93/\$5.00 never produce offspring better off than themselves, except in the rare case of a back or compensatory mutation. Some time after the optimal class of individuals has been lost, the second-best class will suffer the same fate, and so on, leading to a gradual decline in mean fitness.

This phenomenon, now known as Muller's ratchet (Felsenstein 1974), has been subject to numerous theoretical studies with the objective of evaluating the rate at which mean population fitness decays under asexuality (Bell 1988a,b; Birky and Walsh 1988; Charlesworth 1990; Gabriel et al., in press; Haigh 1978; Kondrashov 1982, 1984; Lynch and Gabriel 1990; Maynard Smith 1978; Melzer and Koeslag 1991; Pamilo et al. 1987). But with few exceptions (Bell 1988a,b; Gabriel et al., in press; Lynch and Gabriel 1990; Melzer and Koeslag 1991), these studies have been pursued under the assumption that population size is unaffected by the accumulation of mutations. Although greatly facilitating mathematical analysis, such an assumption seems rather inconsistent with the observation that motivated work on the ratchet-the idea that the longevity of asexual lineages is constrained by mutation accumulation.

Our recent work on Muller's ratchet focuses explicitly on the consequences of mutation accumulation for the survival of asexual lineages (Gabriel et al., in press; Lynch and Gabriel 1990). By reducing the mean absolute fitness of individuals, the 


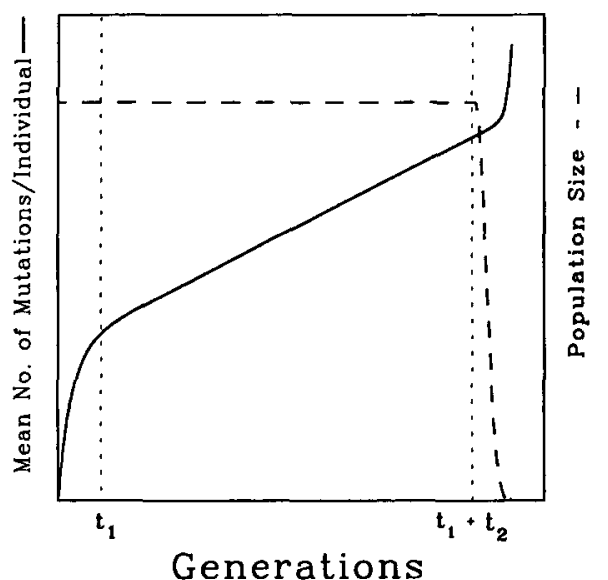

Figure 1. A qualitative description of the temporal pattern of increase in the mean number of deleterious mutations expected in a newly arisen obligately asexual population.

cumulative buildup of mutations is expected to result eventually in a decline in population size. But since chance plays a greater role in smaller populations, once this process begins, each turn of the ratchet increases the subsequent susceptibility of the population to further mutation accumulation. Eventually, random genetic drift completely overwhelms the ability of natural selection to eradicate incoming deleterious mutations, and the population goes extinct. We refer to this synergism between mutation accumulation and random genetic drift as a mutational meltdown.

In this article, we provide an overview of the existing theory on the mutational meltdown. In addition, we introduce some new and simple deterministic results that help clarify the expected dynamics of the mean and variance of numbers of deleterious mutations in an asexual population, an issue that previously has been accessible only by computer simulation. Our attention will be focused entirely on mutation as a source of extinction in obligately asexual lineages. Although mutational meltdowns can occur in sexual species, they are unlikely to be of much significance in populations with effective sizes in excess of a few hundred individuals (Gabriel et al. 1991; Lynch and Gabriel 1990).

\section{Theoretical Results}

Consider a newly arisen asexual lineage, derived, for example, from an ancestral sexual species, and assume that it expands fairly rapidly to its local carrying capacity, $K$. Suppose that adults are capable of producing $R$ progeny each, after which via- bility selection operates prior to reproduction. We first assume that an individual's probability of survival is $W=$ $(1-s)^{n}$, where $s$ is the deleterious effect of each mutation, and $n$ is the number of mutations carried by the individual. Following selection, if the number of surviving zygotes is greater than $K$, density-dependent regulation (independent of genotype) reduces the population back down to $K$; otherwise the new population of reproductive adults is equal to the number of surviving zygotes. Newborns acquire new mutations in a Poisson fashion with a mutation rate per individual of $\mu$.

Mutation accumulation in such a population can be partitioned into three phases (Figure 1). Initially, since all members of the population are recently derived from the same founder individual, there is little variance in the number of mutations among individuals, and selection is relatively ineffective. Gradually, however, the variance in mutation number will approach the level expected under drift-selection-mutation equilibrium. Since the efficiency of selection is directly proportional to the amount of genetic variance, the rate of mutation accumulation declines to a minimum as this second phase is approached. The population then accumulates mutations at a steady rate until the mean viability is reduced to $1 / R$. At that point, the average number of surviving offspring per adult becomes less than one, and the population size begins to decline, initiating the meltdown. Due to the gradual reduction in population size, there is a progressive loss of genetic variance for mutation number during this third phase, and the influence of selection becomes weaker. The rate of mutation accumulation rapidly approaches the mutation rate, driving the population to extinction.

\section{An Analytical Approach}

To a first approximation, the accumulation of deleterious mutations during the first two phases can be described by letting

$$
\bar{n}(t+1) \simeq \bar{n}(t)+\mu-s\left[\lambda \sigma_{n}^{2}(t)+\mu\right],
$$

where $\lambda=1-(1 / K)$ (Gabriel et al., in press; Haigh 1978; Pamilo et al. 1987). In this formulation, the mean number of mutations $(\tilde{n})$ is assumed to be monitored on the adults in each generation that survive selection. Mutation pressure advances the mean number of mutations by $\mu$ per generation, whereas selection reduces the mean in the zygotes by $s\left[\lambda \sigma^{2}{ }_{n}(t)+\mu\right]$. The solution of Equation la requires a second expression for the dynamics of genetic variance in $n$ as influenced by mutation, selection, and random genetic drift. For the model given above, provided $\mu$ is on the order of one, we have found that $n$ can be treated like a quantitative trait, predicting the variance by

$$
\sigma^{2}{ }_{n}(t+1)=\left[\lambda \sigma^{2}{ }_{n}(t)+\mu\right] \Delta_{s}(t) .
$$

In each generation, sampling of adults reduces the variance to a fraction, $\lambda$, of its previous value, while mutation in the progeny restores variance at the rate of $\mu$ per generation. Directional selection for lower $n$ reduces the variance by the fractional amount, $\Delta_{s}(t)$.

With the fitness function given above, $\Delta_{s}(t)$ eventually becomes constant. Thus, during phase two, populations settle into a stochastic equilibrium genetic variance of $n$ determined by the balance between the input by mutation and the loss due to drift and selection. If the average value of $\sigma^{2}{ }_{n}$ observed during phase two in computer simulations is substituted into Equation la, an accurate prediction of the rate of mutation accumulation during that phase is acquired. This validates the structural validity of the model, and indicates that once a mathematical expression for $\Delta_{s}(t)$ is obtained, Equations la,b will provide a useful analytical approximation for predicting the temporal dynamics of $n$.

Unfortunately, after considerable effort, we have still been unable to obtain a general expression for $\Delta_{s}(t)$. Haigh (1978) showed that when populations are effectively infinite in size, $n$ is Poisson distributed, and in this case $\Delta_{s}(t)=1-s$. However, we have found in simulations that the distribution of $n$ can deviate substantially from a Poisson even with $K$ as large as $10^{7}$, especially when $\mu \simeq 1$. This is not surprising since the frequency of individuals in the best class under Haigh's model is $e^{-\mu / s}$, and that of the second-best class is $(\mu / s) e^{-\mu / s}$. With $\mu=1$ and $s=0.025$ (typical parameters that we have employed), these frequencies, $4.2 \times 10^{-18}$ and $1.7 \times$ $10^{-16}$, are orders of magnitude too small to be observed even in enormous populations. Thus, $(1-s)$ is not a generally valid expression for $\Delta_{s}(t)$.

The length of the first phase of mutation accumulation $\left(t_{1}\right)$ is determined by the time that it takes the genetic variance to build up to the asymptotic equilibrium value, $\hat{\sigma}_{n}{ }_{n}$. Through computer simulations, we find that for $K$ larger than a few hundred, the time to reach $95 \%$ of the equilibrium value is approximately $t_{1}=1.5 /\left(s \mu^{\frac{1}{2}}\right)$ generations when $\mu \geq 1$, and approximately $t_{1}=1.5(1$ 
$\left.+e^{-4 \mu}\right) / s$ when $\mu<1$. In both cases, the mean number of mutations per individual at time $t_{1}$ is approximately $\bar{n}\left(t_{1}\right)=1.2 \ln (1$ $+\mu) / s$. Thus, a substantial number of new mutations is expected to accumulate in a population prior to phase two, and at that point, the mean viability will have dropped to approximately $\bar{W}=(1-s)^{\dot{n}\left(\left(_{1}\right)\right.}$. With weak selection $(s<0.1), \bar{W}$ is approximately $1.2 e^{-\mu}$, which is only slightly greater than $e^{-\mu}$, the mean fitness expected in an infinite asexual population (Kimura and Maruyama 1966).

These results show that, when $K$ exceeds a thousand or so, the length of phase one and its effect on mean population fitness are essentially independent of the demographic features of the population ( $K$ and $R$ ). That is not the case for phase two. Consider, for example, the situation in which $K s \ll 1$-i.e., selection is weak relative to drift. The equilibrium level of genetic variance is then very close to the neutral expectation, and hence positively related with the population size,

$$
\hat{\sigma}_{n}^{2} \simeq \mu K
$$

Substituting this into Equation la, it can be seen that, under these conditions, the rate of mutation accumulation during the second phase,

$$
\Delta \bar{n}_{2} \simeq \mu(1-K s),
$$

is only slightly less than the mutation rate, decreasing with increasing $K$.

When selection is strong relative to drift ( $K s \gg 1$ ), the rate of mutation accumulation also declines with increasing $K$, but, provided the genomic mutation rate is $\mu$ $=1.0$ or higher, the dependence on $K$ is very weak (Figure 2). For example, with $\mu$ $=2, \Delta \bar{n}_{2} \simeq \mu / K^{0.09}$, and with $\mu=4, \Delta \bar{n}_{2} \simeq$ $\mu / K^{0.07}$.

The usual view of Muller's ratchet has been that the loss of the best class is due to the random sampling of zygotes (Felsenstein 1974; Haigh 1978; Maynard Smith 1978). However, when the mutation rate is on the order of one per individual, as the data appear to suggest, there is a high probability that the best class will be lost due to mutation pressure alone. Suppose, for example, that there are $X$ individuals in the best class in a population of $K$ reproductive adults. Then provided $X \ll K$, the probability that no members of the next generation are derived from this class is approximately $e^{-x}$ (slightly lower if selection is accounted for). On the other hand, even if $X$ descendants of the best class do make it to the next generation, there is a $\left(1-e^{-\mu}\right)^{X}$ probability that they all will have acquired at least one new mutation. Therefore, the probability of loss of the best class by mutation pressure is at least $P=\left[e\left(1-e^{-\mu}\right)\right]^{X}$ times greater than that due to drift. With $\mu=0.1, P=$ $0.26^{x}$, and drift clearly plays the dominant role in mutation accumulation. But when $\mu=1, P=1.72^{x}$, so the ratchet phenomenon is primarily due to mutation pressure.

With $\mu=1$, the ratchet is expected to turn at the rate of approximately $K^{-0.12}$ per generation, even in very large populations (Figure 2). Thus, the efficiency of selection at removing new mutations is only about $42 \%$ when $K=100,67 \%$ when $K=10^{4}$, and $89 \%$ when $K=10^{6}$. However, when $\mu<1$ and $K$ is large, $\Delta \bar{n}_{2}$ declines rapidly with decreasing $\mu$ (Figure 2). This increase in the efficiency of selection with decreasing $\mu$ is presumably due to the Hill-Robertson effect (Birky and Walsh 1988). When the mutation rate is very low, populations generally consist of only a few mutation-number classes and parallel mutations are less likely. Thus, new mutations are less likely to interfere with each others' elimination.

For the special case in which $\Delta_{s}(t) \simeq(1$ $-s$ ), the dynamics of genetic variance can be solved fairly easily:

$$
\sigma_{n}^{2}(t) \simeq \hat{\sigma}_{n}^{2}(t)\left[1-e^{(\alpha-1) t}\right],
$$

where $\alpha=\lambda(1-s)$, and this allows for an explicit solution for the buildup of the mean number of mutations:

$$
\begin{aligned}
\bar{n}(t) \simeq & \left(\frac{\mu(1-s)}{1+K s}\right) t \\
& +\mu s\left(\frac{\alpha}{1-\alpha}\right)^{2}\left(1-\alpha^{t}\right) .
\end{aligned}
$$

Under these conditions, assuming small $s$ and large $K s$, the equilibrium genetic variance is essentially attained in $t_{1}=3 / \mathrm{s}$ generations, at which point the mean number of mutations per individual is roughly $\mu / s$. Once the second phase has been entered, the expected rate of mutation accumulation is

$$
\bar{n}_{2} \simeq \frac{\mu(1-s)}{1+K s}
$$

which for small $s$ and $K s \gg 1$, reduces to $\Delta \bar{n}_{2} \simeq \mu / K s$. This result is quantitatively consistent with the classical argument that, for populations with large $K$, the rate at which Muller's ratchet turns is directly proportional to $\mu / s$ and inversely proportional to the population size (Bell 1988a; Haigh 1978; Maynard Smith 1978). However, the exact conditions under which Equations 3a,b,c apply are not yet entirely

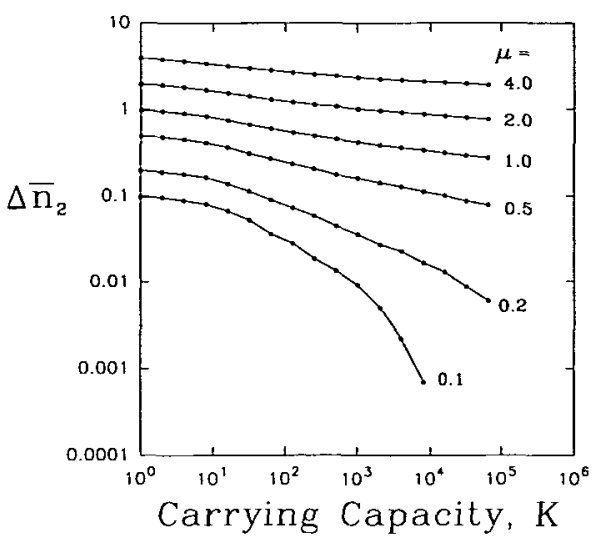

Figure 2. The average rate at which mutations accumulate during phase two $\left(\Delta \bar{n}_{2}\right)$ as a function of population size $(K)$ and the mutation rate $(\mu$, given as insets). The selection intensity is $s=0.025$. Results were obtained by stochastic simulation.

clear. As noted above, the results should be asymptotically correct as $K \rightarrow \infty$ for any $\mu$, but this actually requires very large $K$.

\section{Time to Extinction}

As noted above, the meltdown phase begins when the mean viability has declined to $1 / R$. Thus, since the mean viability after phase one is expected to be approximately $e^{-\mu}$, we expect the final phase of mutation accumulation to begin after approximately

$$
t_{2}=-\frac{\ln \left(R e^{-\mu}\right)}{\Delta \bar{n}_{2} \ln (1-s)}
$$

generations have elapsed in phase two. The expected time to the initiation of the meltdown is then approximately $t_{1}+t_{2}$ generations. Since $t_{1}$ and $\Delta \bar{n}_{2}$ are both independent of $R$, this suggests that the mean time to extinction should increase linearly with the natural logarithm of the reproductive rate. Our results from computer simulations show that this is indeed the case (Figure 3 ).

Figure 3 also shows that the effect of population size on the extinction time increases with the logarithm of $K$, but at a surprisingly slow rate. For example, a 64 fold increase in $K$ from 32 to 2,048 only increases the time to extinction by about $50 \%$. When the reproductive rate is low, the mean absolute times to extinction are very low for populations of all sizes. Thus, as we have emphasized elsewhere (Lynch and Gabriel 1990), for organisms that reproduce by binary fission, we expect to observe characteristic extinction times that are largely independent of population size. This prediction is qualitatively consistent with observations on laboratory cultures of ciliates and fibroblasts (Bell 1988b; Lynch and Gabriel 1990). Claims have of- 


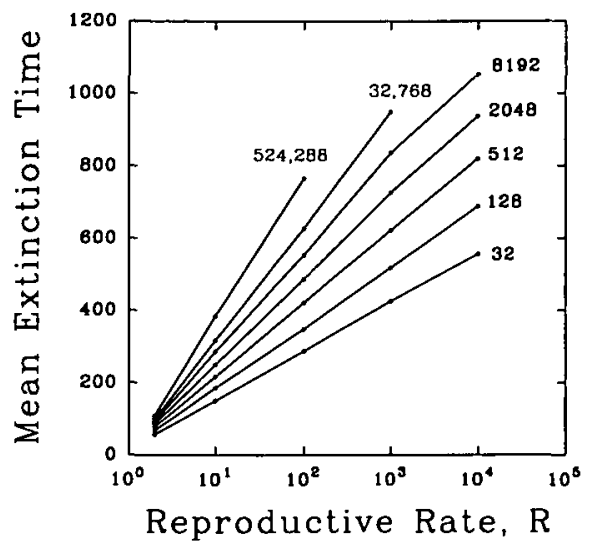

Figure 3. The mean time to extinction for obligately asexual populations as a function of the reproductive rate $(R)$. Data obtained by computer simulation are given for populations with various carrying capacities ( $K$, given adjacent to the plotted lines). The mutation rate, $\mu$, was assumed to be one per zygote per generation, and each mutation was assumed to reduce the viability of zygotes by $s=0.025$.

ten been made that such results are a consequence of "programmed cell death," but empirical data seem to be consistent with a gradual decline in cell division potential throughout the period of culture (Bell 1988b; Lynch and Gabriel 1990).

Populations with larger $K$ have an advantage in that the length of the meltdown phase $\left(t_{3}\right)$ will be longer, but this advantage is not expected to be very large. Since the length of the meltdown phase depends on the time it takes the mean viability to decline from $1 / R$ to approximately $1 / R K$, we expect $t_{3}$ to increase only with the logarithm of $K$. Our computer simulations verify that this is indeed the case. Moreover, although the ratchet continues to turn at the approximate rate $\Delta \bar{n}_{2}$ in the early stages of phase three, until the adult population size has been reduced enough to have a significant influence on the genetic variance, shortly thereafter $\Delta \bar{n}$ will approach the mutation rate as drift completely overwhelms selection. Consequently, $t_{3}$ is generally $\ll\left(t_{1}+t_{2}\right)$. Thus, when $\Delta \tilde{n}_{2}$ is known from computer simulations, the quantity $\left(t_{1}+t_{2}\right)$ provides a close approximation to the mean extinction time.

When mutation pressure is high, the time to extinction is expected to be only weakly dependent on $K$, since with $K s \gg 1$, both $t_{1}$ and $t_{2}$ are nearly independent of $K$. On the other hand, when the mutation pressure is low, the rate of mutation accumulation in the second phase is inversely proportional to $K$ (Figure 2), and this phase completely dominates the total period of mutation accumulation. So when $\mu<0.1$, the extinction time is expected to be pro- portional to $K$. This can be seen most easily for the situation in which Equations 3a,b,c apply by substituting Equation 3c into Equation 4. Assuming small $s$ and $K s$ $\gg 1$, we obtain $t_{2} \simeq K \ln \left(R e^{-\mu}\right) / \mu$, which also shows that under these conditions, $s$ has a negligible influence on the mean extinction time.

The results in Figure 3 provide quantitative insight into how long obligately asexual lineages are expected to withstand the constant onslaught of deleterious mutations. In our simulations, there are no sources of mortality other than the viability mutations themselves, the population is assumed to be initiated with no deleterious mutations, and the mutations do not have negative effects on either $R$ or $K$. Thus, the plotted values are clear upper limits to the extinction time for the mutation parameters assumed in the simulations. To the extent that these parameter estimates are reasonable, this implies that obligately asexual lineages are very unlikely to persist for more than a few thousand generations unless $K$ is large enough to permit ample opportunity for compensatory or back mutations prior to the mutational meltdown. This conclusion is quite consistent with existing molecular data, most of which indicate that parthenogenetic lineages of plants and animals usually are not removed by much more, and often considerably less, than $10^{4}$ generations from their sexual ancestors (Bell 1982; Lynch 1984; Maynard Smith 1992).

Another interesting feature of the mutational meltdown is the rather low variance in extinction time expected among replicate populations. For example, in Figure 3 , all but the populations with $K=32$ have coefficients of variation of extinction time less than 0.1 throughout the full range of $R$, and these decline with increasing $R$ and $K$. Since qualitatively similar results have been found with different demographic models (Gabriel et al., in press; Lynch and Gabriel 1990), this provides further support for the idea that clonal lineages should have fairly characteristic extinction times, determined primarily by the mutation parameters.

Classical results on Muller's ratchet, obtained under the assumption of an effectively infinite population size, led to the reasonable suggestion that the rate of mutation accumulation should increase with decreasing $s$ (Haigh 1978). Although true, this does not convey an accurate description of the influence of mutation accumulation on mean population fitness. The lower rate of accumulation of mutations with large $s$ can be more than offset by the greater damage they do to the population per turn of the ratchet (Gabriel et al., in press). Consequently, as $s$ increases, the mean time to extinction declines until a critical value, $s^{*}$, is exceeded. Beyond this point, the ratchet turns very slowly, until with $s=1$ (lethal mutations), it cannot turn at all, and extinction only occurs in the unlikely event that every member of the population incurs a mutation in the same generation.

The value of $s$ that minimizes the extinction time arises at the point where the decline in the rate of the ratchet is balanced by the increase in the damage per turn of the ratchet. Beyond this point, the reduction in the rate of the ratchet more than offsets the damage per turn. Thus, any factors that increase the efficiency of selection are expected to shift the critical value of $s$ to smaller values. As noted above, large $K$ and small $\mu$ both enhance the efficiency of selection, and we have found that such conditions do indeed lead to smaller values of $s^{*}$ (Gabriel et al., in press).

Except in the case of very small populations under high mutation pressure, the critical value $s^{*}$ is on the order of 0.1 or less (Gabriel et al., in press). This raises interesting questions about the evolution of systems for repairing mutations and/or DNA damage. If uncorrected mutations and DNA damage usually cause a reduction in viability in excess of $10 \%$, their repair has to be efficient enough to reduce $s$ below a threshold value before an improvement in the longevity of the lineage can be realized (Figure 4). Since asexual lineages are reproductively isolated from each other, this suggests the possibility that lineages with poor proofreading capacity and/or damage repair mechanisms can displace others for which the inherited effects of mutations are lower. By computer simulation, we have shown that lineage sorting can indeed override the individual advantages of low $s$ (Gabriel et al., in press). Thus, unless the effects of all mutations are less than the critical value of $s$, obligate asexuality can lead to the evolution of genetic systems that insure that the individual effects of mutations are quite high.

Up to now, we have been focusing on aspects of mutation accumulation when the fitness function is $W=(1-s)^{n}$. Under this model, the expected rate of mutation accumulation eventually settles into the steady-state $\Delta \bar{n}_{2}$ since successive clicks of the ratchet reduce fitness by the same amount $(1-s)$. With a more or less steep 


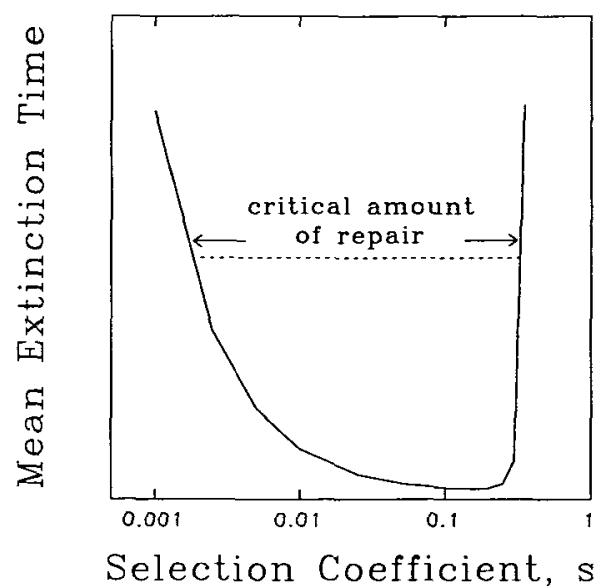

Figure 4. The qualitative relationship between the expected longevity of an asexual lineage and the selection coefficient. The horizontal line denotes the degree to which the effect of a mutation has to be reduced in order to increase the longevity of the lineage.

fitness function, the rate of mutation accumulation can decrease or increase progressively.

To evaluate the extent to which a synergistic fitness function might be able to prevent the mutational meltdown, we considered the linear function $W=1-s n$. When $s n$ is small, this yields essentially the same fitness as $W=(1-s)^{n}$. But for a given $n$, fitness is always lower with the linear function, and with $n \geq 1 / s, W=0$ with this model. Figure 5 shows that when $R$ is small, the linear function leads to more rapid extinction, but once $R$ exceeds a threshold value, $I_{E}$ increases very rapidly with increasing $R$.

There is a simple explanation for why a synergistic fitness function can prevent a mutational meltdown in a high-fecundity population. Recall that the meltdown phase is entered when adults produce an average of less than one surviving offspring per capita. Let $W^{*}$ be the fitness of individuals in the class just prior to the $n$ that gives $W=0$ (with the linear fitness function, $W^{*}$ $=s$ ). The lowest possible per capita progeny production is then $R W^{*} e^{-\mu}$, where $e^{-\mu}$ is the probability that a newborn does not incur any new mutations. Thus, a population will not enter the meltdown phase if $R$ exceeds $e^{\mu} / W^{*}$. With a linear fitness function, $\mu=1$, and $s=0.025$, this critical value is $R=109$. With large $K$, it is extremely unlikely that the entire population will decline to fitness $W^{*}$, so this is a conservatively high estimate of the reproductive rate necessary to avoid the meltdown, as can be seen in Figure 5 . On the other hand, when $K$ is small, there can still be an appreciable probability of extinction in phase two, even if the meltdown phase cannot be entered.

In reality, a synergistic fitness function may be much less effective at promoting population longevity than the results in Figure 5 suggest. It seems extremely unlikely that all mutations have a constant effect of $s$ as in our idealized models. More likely, the effects of new mutations are distributed continuously with mean effect, $s$, and minimum effect, $s_{m}$, in which case the ratchet will continue until $W^{*}$ declines to $s_{m}$ (rather than $s$ ). In this case, the $R$ required to escape the meltdown is $e^{u} / s_{m}$, so if $s_{m}$ is very close to zero, a synergistic fitness function cannot prevent a mutational meltdown (Butcher D, unpublished manuscript).

\section{Discussion}

Several attempts have been made recently to understand the evolutionary advantages of diploidy, with the major conclusion being fairly intuitive-that diploidy serves to mask the effects of deleterious mutations (Bengtsson 1992; Kondrashov and Crow 1991; Otto and Goldstein 1992; Perrot et al. 1991). It is perhaps notable that the theoretical work done on this subject has been confined to sexual species, as it is difficult to see how the main conclusion can be extended to asexual species. Since deleterious mutations are expected to accumulate to a much greater extent in asexual species than in their sexual counterparts, the masking effect would be expected to be especially beneficial in the absence of segregation and recombination. Yet most of the world's abundant asexual organisms (bacteria, algae, fungi) are haploid, and many species with alterations of asexual and sexual generations remain haploid during the asexual phase.

A possible explanation for this observation is provided by our argument that lineage sorting in asexual organisms can lead to the long-term proliferation of lineages with high sensitivity of individuals to mutations. If the individual effects of all mutations were less than $s^{*}$, diploidy would provide no advantage to an asexual organism if the mutations had additive effects at each locus. The $50 \%$ reduction in the effect of the mutation by masking would be essentially offset by the twofold increase in the genomic mutation rate. If, on the other hand, many mutations have effects in excess of $s^{*}$, the reduction in the effect of the mutation under diploidy, combined with the doubling of the mutation rate, can lead to a reduction in the ex-

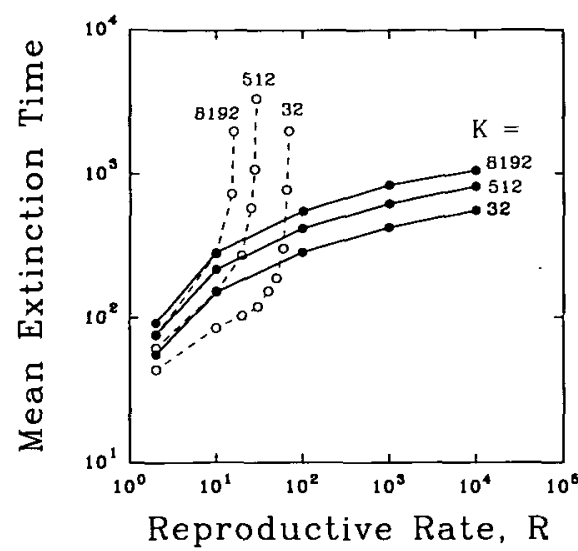

Figure 5. The mean time to extinction for obligately asexual populations as a function of the reproductive rate, $R$, and the carrying capacity, $K$. Results are given for exponential (solid lines, closed circles) and linear (dotted lines, open circles) fitness functions with $s=$ 0.025 . The mutation rate, $\mu$, was assumed to be one per zygote per generation.

pected longevity of the lineage. This argument is, of course, weakened if most new mutations are highly recessive, but it cannot be completely invalid unless mutant alleles are completely recessive. Neither of these conditions are compatible with the existing data (Mukai 1979). In any event, haploidy does not simply enhance the impact of mutations at the individual level by the elimination of masking. It also removes a major mechanism, available to all known diploids, for coping with DNA damage-the repair of double-strand breaks off a homologous chromosome. Thus, haploidy ensures that the effects of damage will usually be lethal. It seems hard to escape the conclusion that haploidy has evolved in asexual lineages despite the advantage of diploidy at the individual level.

Similar arguments may also bear on the seemingly low rate of mutational degradation of organelle genomes, an issue that has been difficult to explain on theoretical grounds (Bell 1988a; Gabriel et al., in press; Takahata and Slatkin 1983). Although a detailed understanding of the transmission genetics of organelle genomes is still lacking, indirect evidence suggests that the effective number of mitochondria transmitted by an individual is usually less than a few hundred, and in some cases as few as three or four (Koehler et al. 1991; Rand and Harrison 1986; Satoh and Kuroiwa 1991; Solignac et al. 1987). Since an individual's organelles are usually inherited entirely or nearly entirely from one parent, the "population" structure consists of very small isolated demes. So conditions seem to be optimal for the operation of Muller's ratchet within each isolated lineage. Yet mitochondria and chloroplasts and many 
of their hosts have been in existence for $\sim 10^{9}$ years.

Like other long-lived asexual lineages, animal mitochondria appear to maximize individual sensitivity to mutations and DNA damage. First, they are haploid. Second, and even more notably, they have completely lost all of the genes for proofreading and break-repair enzymes, both of which were almost certainly present in their bacterial ancestors. Third, all of the transfer RNA genes and the ribosomal RNA genes, which are normally present in multiple copies in nuclear genomes, exist as single copies. Under these conditions, any important functional change in a tRNA anticodon or amino-acid loading site would be expected to be completely debilitating, contrary to the situation in the nucleus where some compensation is possible because of copy redundancy. Similar arguments can be made for the rRNAs. Fourth, because mitochondrial genomes are housed in small vessels that are the sites of intense metabolic activity, they are often exposed to exceptionally mutagenic conditions. When a mutation in a proteincoding gene causes a significant increase in the oxidative conditions in a mitochondrion, the probability of subsequent mutations can be magnified substantially, leading to a "runaway" mutation process that is now thought to be a major cause of aging and debilitative disease (Wallace 1992). Fifth, a common type of mutation in mitochondria is the deletion of a major segment of the genome (Holt et al. 1988). Due to the near complete lack of spacer DNA in the mitochondrial genome, this almost always leads to loss of metabolic function. But for reasons that are not well understood, such mutant DNAs often have a replicative advantage that can drive them toward fixation, leading to death of the host (Shoubridge et al. 1990).

All of these processes seem to ensure that when a deleterious mutation arises in a mitochondrion, its effects will not just be accepted, but often magnified to the extent that it will easily be eliminated from the population. Host individuals may be lost regularly in this process, but entire host lineages would not be imperiled by gradual and cumulative mutation accumulation in their asexual symbionts. Thus, the extreme longevity of organelle lineages may be due not to their invulnerability to mutations and DNA damage, but to their extreme sensitivity to such effects.

\section{References}

Bell G, 1982. The masterpiece of nature: the evolution and genetics of sexuality. Berkeley, California: University of California Press.

Bell G, 1988a. Recombination and the immortality of the germ line. J Evol Biol 1:67-82.

Bell G, 1988b. Sex and death in the protozoa. New York: Cambridge University Press.

Bengtsson BO, 1992. Deleterious mutations and the origin of the meiotic ploidy cycle. Genetics 131:741744.

Birky CW Jr and Walsh JB, 1988. Effects of linkage on rates of molecular evolution. Proc Natl Acad Sci USA 85:6414-6418.

Charlesworth B, 1990. Mutation-selection balance and the evolutionary advantage of sex and recombination. Genet Res (Cambridge) 55:199-221.

Charlesworth B, Charlesworth D, and Morgan MT, 1990. Genetic loads and estimates of mutation rates in highly inbred plant populations. Nature 347:380-382.

Crow JF and Simmons MJ, 1983. The mutation load in Drosophila. In: The genetics and biology of Drosophila Vol. 3c (Ashburner M, Carson HP, and Thompson JN Jr, eds). New York: Academic Press; 1-35.

Felsenstein J, 1974. The evolutionary advantage of recombination. Genetics 78:737-756.

Gabriel W, Bürger R, and Lynch M, 1991. Population extinction by mutational load and demographic stochasticity. In: Species conservation: a population-biological approach (Seitz $A$ and Loeschke V, eds). Basel, Switzerland: Birkhäuser Verlag; 49-59.

Gabriel W, Lynch $M$, and Bürger $R$, in press. Muller's ratchet and mutational meltdowns. Evolution.

Haigh J, 1978. The accumulation of deleterious genes in a population. Theor Pop Biol 14:251-267.

Haldane JBS, 1937. The effect of variation on fitness. Am Nat 71:337-349.

Holt IJ, Harding AE, and Morgan-Hughes JA, 1988. Deletions of mitochondrial DNA in patients with mitochondrial myopathies. Nature 331:717-719.

Houle D, Hoffmaster DK, Assimacopoulos S, and Charlesworth B, 1992. The genomic mutation rate for fitness in Drosophila. Nature 359:58-60.

Kimura $M$ and Maruyama T, 1966. The mutation load with epistatic gene interactions in fitness. Genetics 54 1337-1351.

Kimura M, Maruyama T, and Crow JF, 1963. The mu tation load in small populations. Genetics 48:1303-1312.
Koehler CM, Lindberg GL, Brown DR, Beitz DC, Freeman AE, Mayfield JE, and Myers AM, 1991. Replacement of bovine mitochondrial DNA by a sequence variant within one generation. Genetics 129:247-255.

Kondrashov AS, 1982. Selection against harmful mutations in large sexual and asexual populations. Genet Res (Cambridge) 40:325-332.

Kondrashov AS, 1984. Deleterious mutations as an evolutionary factor. Genet Res (Cambridge) 44:199-217.

Kondrashov AS, 1988. Deleterious mutations and the evolution of sexual reproduction. Nature 334:435-440.

Kondrashov AS and Crow JF, 1991. Haploidy or diploidy: which is better? Nature 351:314-315.

Lynch M, 1984. Destabilizing hybridization, generalpurpose genotypes, and geographic parthenogenesis. Quart Rev Biol 59:257-290.

Lynch $\mathrm{M}$ and Gabriel W, 1990. Mutation load and the survival of small populations. Evolution 44:1725-1737.

Maynard Smith J, 1978. The evolution of sex. New York: Cambridge University Press.

Maynard Smith J, 1992. Age and the unisexual lineage. Nature 356:661-662.

Melzer AL and Koeslag JH, 1991. Mutations do not accumulate in asexual isolates capable of growth and extinction-Muller's ratchet reexamined. Evolution 45: 649-655.

Mukai T, 1979. Polygenic mutation. In: Quantitative genetic variation (Thompson JN Jr and Thoday JM, eds). New York: Academic Press; 177-196.

Muller HJ, 1964. The relation of recombination to mutational advance. Mutation Res 1:2-9.

Otto SP and Goldstein DB, 1992. Recombination and the evolution of diploidy. Genetics 131:745-751.

Pamilo P, Neí M, and Li WH, 1987. Accumulation of mutations in sexual and asexual populations. Genet Res (Cambridge) 49:135-146.

Perrot V, Richerd S, and Valéro M, 1991. Transition from haploidy to diploidy. Nature 351:315-317.

Rand DM and Harrison RG, 1986. Mitochondrial DNA transmission genetics in crickets. Genetics 114:955970.

Satoh $M$ and Kuroiwa T, 1991. Organization of multiple nucleoids and DNA molecules in mitochondria of a human cell. Exp Cell Res 196:137-140.

Shoubridge EA, Karpati G, and Hastings KEM, 1990. Deletion mutants are functionally dominant over wildtype mitochondrial genomes in skeletal muscle fiber segments in mitochondrial disease. Cell 62:43-49.

Solignac M, Génermont J, Monnerot M, and Mounolou JC, 1987. Drosophila mitochondrial genetics: evolution of heteroplasmy through germ line cell divisions. Genetics 117:687-696.

Takahata $\mathrm{N}$ and Slatkin M, 1983. Evolutionary dynamics of extranuclear genes. Genet Res (Cambridge) 42 : 258-277.

Wallace DC, 1992. Mitochondrial genetics: a paradigm for aging and degenerative diseases? Science 256:628632. 